\title{
A best possible result for the square of a 2-block to be hamiltonian
}

\author{
Jan Ekstein ${ }^{\mathrm{a}}$, Herbert Fleischner ${ }^{\mathrm{b}}$ \\ a Department of Mathematics and European Centre of Excellence NTIS - New Technologies for the Information Society, \\ Faculty of Applied Sciences, University of West Bohemia, Pilsen, Technická 8, 30614 Plzeň, Czech Republic \\ ${ }^{\mathrm{b}}$ Institute of Logic and Computation, Algorithms and Complexity Group, Technical University of Vienna, Favoritenstrasse 9-11, \\ 1040 Wien, EU, Austria
}

\section{A R T I C L E I N F O}

\section{Article history:}

Received 4 June 2019

Received in revised form 22 June 2020

Accepted 7 September 2020

Available online $\mathrm{xxxx}$

\section{Keywords:}

Square of graphs

Hamiltonian cycles

\section{A B S T R A C T}

It is shown that for any choice of four different vertices $x_{1}, \ldots, x_{4}$ in a 2-block $G$ of order $p>3$, there is a hamiltonian cycle in $G^{2}$ containing four different edges $x_{i} y_{i}$ of $E(G)$ for certain vertices $y_{i}, i=1,2,3,4$. This result is best possible.

(c) 2020 Elsevier B.V. All rights reserved.

\section{Introduction}

As for standard terminology, we refer to the book by Bondy and Murty, [2], and to the papers quoted in the references.

The square of a graph $G$, denoted $G^{2}$, is the graph obtained from $G$ by joining any two nonadjacent vertices which have a common neighbor, by an edge. Fairly recent development in hamiltonian graph theory has shown a resurgence of interest in hamiltonian cycles and paths in the square of 2-connected graphs (which we call 2-blocks for short). In particular, short proofs have been found for two results of the second author of the present paper, [10,11]. And more recently, in [1] the authors develop algorithms which are linear in $|E(G)|$ and produce a hamiltonian cycle, a hamiltonian path joining arbitrary vertices $u$ and $v$ respectively, in $G^{2}$. Moreover, they develop an algorithm running in $O\left(|V(G)|^{2}\right)$ time and producing cycles of arbitrary length from 3 to $|V(G)|$.

Also very recently it was shown in [3] and [8] that a 2-block has the $\mathcal{F}_{4}$ property; that is, given vertices $x_{1}, x_{2}, x_{3}, x_{4}$ in the 2-block $G$, there is a hamiltonian path in $G^{2}$ joining $x_{1}$ and $x_{2}$ and traversing distinct edges $x_{3} y_{3}$ and $x_{4} y_{4}$ of $G$ (see Theorem 7). The proof of this result is very long and is based on techniques developed by Fleischner in [5-7] and by Fleischner and Hobbs in [9]. It remains to be shown whether one can find a much shorter proof of this result. However, this result will be of importance in the proof of the main result of the current paper.

We start with a definition.

Definition 1. A graph $G$ is said to have the $\mathcal{H}_{k}$ property if for any given vertices $x_{1}, \ldots, x_{k}$ there is a hamiltonian cycle in $G^{2}$ containing distinct edges $x_{1} y_{1}, \ldots, x_{k} y_{k}$ of $G$.

We note in passing that $G$ having the $\mathcal{F}_{4}$ property implies that $G$ has the $\mathcal{H}_{3}$ property; clearly, choose $x_{1}, x_{2}, x_{3}$ arbitrarily and a different $x_{4}$ adjacent to some $x_{1}$ for $i \in\{1,2,3\}$ in $G$, say $i=1$. A hamiltonian path in $G^{2}$ joining $x_{1}$ and $x_{4}$ and containing edges $x_{2} y_{2}$ and $x_{3} y_{3}$ of $G$ yields a hamiltonian cycle containing these two edges of $G$ and $x_{1} x_{4}$ which lies also in $G$.

E-mail addresses: ekstein@kma.zcu.cz (J. Ekstein), fleischner@ac.tuwien.ac.at (H. Fleischner). 
The main result of this paper is the following.

Theorem 2. Given a 2-block $G$ on at least four vertices, then $G$ has the $\mathcal{H}_{4}$ property, and there are 2-blocks of arbitrary order greater than 4 without the $\mathcal{H}_{5}$ property.

This theorem and the $\mathcal{F}_{4}$ property of 2-blocks are key to describe the most general block-cut vertex structure a graph $G$ may have in order to guarantee that $G^{2}$ is hamiltonian, hamiltonian connected, respectively. This will be done in follow-up papers.

Moreover Theorem 2 gives the positive answer to Conjecture 5.4 stated in [4] as an immediate corollary.

Corollary 3. Let $G$ be a connected graph such that its block-cutvertex graph $b c(G)$ is homeomorphic to a star in which the center $c$ corresponds to a block $B_{c}$ of $G$. If $B_{c}$ contains at most 4 cutvertices, then $G^{2}$ is hamiltonian.

\section{Preliminaries}

However, before proving Theorem 2 we mention several concepts and results which we need to make use of, and we prove a lemma.

A graph $G$ is an edge-critical block, if $\kappa(G)=2$ and $\kappa(G-e)=1$ for any edge $e$ of $G$. Let $D(G)$ be the set of edges $u v$ where both $d_{G}(u) \geq 3$ and $d_{G}(v) \geq 3$. If $D(G)=\emptyset$, then every edge of $G$ is incident to a vertex of degree 2 ; we call such a graph a DT-graph.

Theorem 4 ([6]). Let $G$ be an edge-critical block. Then exactly one of the following two statements is true:

(1) $G$ is a DT-block.

(2) There is an edge $f$ in $D(G)$ such that at least one of the endblocks of $G-f$ is a DT-block.

The basic result about hamiltonicity of the square of a 2-block is given by the following theorem.

Theorem 5 ([7]). Suppose $v$ and $w$ are two arbitrarily chosen vertices of a 2-block $G$. Then $G^{2}$ contains a hamiltonian cycle $C$ such that the edges of $C$ incident to $v$ are in $G$ and at least one of the edges of $C$ incident to $w$ is in G. Furthermore, if $v$ and $w$ are adjacent in $G$, then these are three different edges.

Let $\mathrm{bc}(G)$ denote the block-cutvertex graph of $G$. Blocks corresponding to leaves of $\mathrm{bc}(G)$ are called endblocks. Note that a block in a graph $G$ is either a 2-block or a bridge of $G$. The graph $G$ is called blockchain if bc $(G)$ is a path. Let $G$ be a blockchain. We denote its blocks $B_{1}, B_{2}, \ldots, B_{k}$ and cutvertices $c_{1}, c_{2}, \ldots, c_{k-1}$ such that $c_{i} \in V\left(B_{i}\right) \cap V\left(B_{i+1}\right)$, for $i=1,2, \ldots, k-1$. A blockchain $G$ is called trivial, if $E(\mathrm{bc}(G))=\emptyset$, otherwise it is called non-trivial. Note that only $B_{1}$ and $B_{k}$ are endblocks of a non-trivial blockchain $G$. An inner block is a block of $G$ containing exactly 2 cutvertices. An inner vertex is a vertex in $G$ which is not a cutvertex of $G$.

The first author proved in [4] the following theorem dealing hamiltonicity of the square of a blockchain graph.

Theorem $6([4])$. Let $G$ be a blockchain and let $u_{1}, u_{2}$ be arbitrary inner vertices which are contained in different endblocks of $G$.

Then $G^{2}$ contains a hamiltonian cycle $C$ such that, for $i=1,2$,

- if $u_{i}$ is contained in a 2-block, then both edges of $C$ incident with $u_{i}$ are in $G$, and

- if $u_{i}$ is not contained in a 2-block, then exactly one edge of $C$ incident with $u_{i}$ is in $G$.

Let $G$ be a connected graph. By a $u v$-path we mean a path from $u$ to $v$ in $G$. If a $u v$-path is hamiltonian, we call it a $u v$-hamiltonian path. Let $A=\left\{x_{1}, x_{2}, \ldots, x_{k}\right\}$ be a set of $k(\geq 3)$ distinct vertices in $G$. An $x_{1} x_{2}$-hamiltonian path in $G^{2}$ which contains $k-2$ distinct edges $x_{i} y_{i} \in E(G), i=3, \ldots, k$, is said to be $\mathcal{F}_{k}$. A graph $G$ is said to have the $\mathcal{F}_{k}$ property if, for any set $A=\left\{x_{1}, x_{2}, \ldots, x_{k}\right\} \subseteq V(G)$, there is an $\mathcal{F}_{k} x_{1} x_{2}$-hamiltonian path in $G^{2}$.

Theorem 7 ([8]). Let $G$ be a 2-block. Then $G$ has the $\mathcal{F}_{4}$ property.

A graph $G$ is said to have the strong $\mathcal{F}_{3}$ property if, for any set of 3 vertices $\left\{x_{1}, x_{2}, x_{3}\right\}$ in $G$, there is an $x_{1} x_{2}$-hamiltonian path in $G^{2}$ containing distinct edges $x_{3} z_{3}, x_{i} z_{i} \in E(G)$ for a given $i \in\{1,2\}$. Such an $x_{1} x_{2}$-hamiltonian path in $G^{2}$ is called a strong $\mathcal{F}_{3} x_{1} x_{2}$-hamiltonian path.

Theorem 8 ([8]). Every 2-block has the strong $\mathcal{F}_{3}$ property.

The following lemma is frequently used in the proofs below.

Lemma 9. Let $G$ be a non-trivial blockchain. We choose

- $c_{0} \in V\left(B_{1}\right), c_{k} \in V\left(B_{k}\right)$ which are not cutvertices;

- $u_{i} \in V\left(B_{i}\right)$ (if any) which is not a cutvertex and $v_{i} \in V\left(B_{i}\right)$ such that $u_{i} \neq v_{i}, u_{1} \neq c_{0}$ and $u_{k} \neq c_{k}$, for $i=1,2, \ldots, k$. 
Then $G^{2}$ contains a $c_{0} c_{k}$-hamiltonian path $P$ such that there exist distinct edges $u_{i} u_{i}^{\prime} v_{i} v_{i}^{\prime} \in E\left(B_{i}\right) \cap E(P)$ (if $u_{i}$ exists), $i=1,2, \ldots, k$.

Proof. If $B_{i}$ is 2-connected, then let $P_{i}$ be an $\mathcal{F}_{4} c_{i-1} c_{i}$-hamiltonian path in $B_{i}^{2}$ containing 2 distinct edges $u_{i} u_{i}^{\prime}, v_{i} v_{i}^{\prime} \in E\left(B_{i}\right)$ for $v_{i} \notin\left\{c_{i-1}, c_{i}\right\}$ by Theorem 7; and let $P_{i}$ be a strong $\mathcal{F}_{3} c_{i-1} c_{i}$-hamiltonian path in $B_{i}^{2}$ containing 2 distinct edges $u_{i} u_{i}^{\prime}, v_{i} v_{i}^{\prime} \in E\left(B_{i}\right)$ for $v_{i} \in\left\{c_{i-1}, c_{i}\right\}$ by Theorem 8, respectively.

If $B_{i}=c_{i-1} c_{i}$, then we set $P_{i}=B_{i}$. Note that in this case $u_{i}$ does not exist and $v_{i} \in\left\{c_{i-1}, c_{i}\right\}$.

Then $P=\cup_{i=1}^{k} P_{i}$ is a $c_{0} c_{k}$-hamiltonian path in $G^{2}$ as required.

The concept of EPS-graphs plays a central role in proofs of hamiltonicity in the square of a DT-graph (see [5]). We use this concept also in one part of the proof of Theorem 2. Let $G$ be a graph. An EPS-graph is a spanning connected subgraph $S$ of $G$ which is the edge-disjoint union of an Eulerian graph $E$ (which may be disconnected) and a linear forest $P$. For $S=E \cup P$, let $d_{E}(v), d_{P}(v)$ denote the degree of $v$ in $E, P$, respectively.

Fleischner and Hobbs introduced in [9] the concept of $W$-soundness of a cycle. Let $W$ be a set of vertices of $G$. A cycle $K$ is called $W$-maximal if $\left|V\left(K^{\prime}\right) \cap W\right| \leq|V(K) \cap W|$ for any cycle $K^{\prime}$ of $G$. Let $K$ be a cycle of $G$ and let $W$ be a set of vertices of $G$. A blockchain $P$ of $G-K$ is a $W$-separated $K$-to-K blockchain based on vertex $x$ if a vertex of $W$ is a cut vertex of $P$, both endblocks $B$ and $B^{\prime}$ of $P$ include vertices of $K, V(B) \cap V(K)=\{x\}$, no vertex of $K$ is a cutvertex of $P$, and $(V(P) \cap V(K))-\{x\} \subseteq V\left(B^{\prime}\right)$. For a given path $p=v_{1}, v_{2}, \ldots, v_{n-1}, v_{n}$ we let $F(p)=v_{1}, L(p)=v_{n}$.

Definition 10. A cycle $K$ in $G$ is $W$-sound if it is $W$-maximal, $|W|=5$ and the following hold:

(1) $|V(K) \cap W| \geq 4$; or

(2) $|V(K) \cap W|=3$ and the following situation does not prevail; there are two $W$-separated $K$-to- $K$ blockchains $P$ and $Q$ of $G-K$ based on a vertex $w$ of $W$ such that $V(P) \cap V(Q)=\{w\}$ and if $p$ is a shortest path in $P$ from $w$ to a vertex of $K$ different from $w$ and $q$ is the same for $Q$, then there is a subsequence $w, w^{\prime}, L(p), L(q), w^{\prime \prime}, w$ of $K$ where $w^{\prime}$ and $w^{\prime \prime}$ are in $W-\{w\}$; or

(3) $|V(K) \cap W|=2$ and the following situation does not prevail; there are three $W$-separated $K$-to- $K$ blockchains $P_{1}, P_{2}$ and $P_{3}$ of $G-K$ based on a single vertex $a$ of $V(K)-W$, such that $V\left(P_{i}\right) \cap V\left(P_{j}\right)=\{a\}$ whenever $i$ and $j$ are distinct elements of $\{1,2,3\}$, and if $p_{i}$ is a shortest path in $P_{i}$ from $a$ to a vertex of $K$ different from $a$ for each $i \in\{1,2,3\}$, then there is a subsequence $a, w^{\prime}, L\left(p_{1}\right), L\left(p_{2}\right), L\left(p_{3}\right), w^{\prime \prime}, a$ of $K$ where $\left\{w^{\prime}, w^{\prime \prime}\right\}=V(K) \cap W$.

We observe that Definition 10 is basically the content of Lemma 1 in [9]. That is, said lemma guarantees that for every choice $W \subseteq V(G)$ with $|W|=5$ in a 2-block $G$ of order at least 5 , there is a $W$-sound cycle in $G$.

Theorem 11 ([9]). Let $G$ be a 2-block and $W$ a set of five distinct vertices in $G$, and let $K$ be a $W$-sound cycle in $G$. Then there is an EPS-graph $S=E \cup P$ of $G$ such that $K \subseteq E$ and $d_{P}(w) \leq 1$ for every $w \in W$.

\section{Proof of Theorem 2}

Proof. First we prove that $G$ has the $\mathcal{H}_{4}$ property. We proceed by contradiction supposing that $|V(G)|+|E(G)|$ is minimal. It follows that $G$ is an edge-critical block and in particular $|V(G)| \geq 5$. We distinguish cases by the number of edges in $D(G)$. The reader is advised to draw figures where he/she deems it necessary to follow our case distinctions.

Case 1. $|D(G)|>0$. By Theorem 4, let $f=x^{\prime} x \in D(G)$ be an edge where both $d_{G}\left(x^{\prime}\right) \geq 3$ and $d_{G}(x) \geq 3$. Then $G-f$ is a blockchain and both endblocks $B^{\prime}, B$ of $G-f$ are 2-blocks. Set $X=\left\{x_{1}, x_{2}, x_{3}, x_{4}\right\}$. Without loss of generality assume that $|X \cap(V(B)-y)| \leq 2$ (otherwise we consider $B^{\prime}$ instead of $B$ ); i.e., at most $x_{1}, x_{2} \in V(B)-y$, say, where $x, y \in V(B)$ and $y$ is a cutvertex of $G-f$. We distinguish the following 3 subcases.

Subcase 1.1: $|X \cap(V(B)-y)|=2$; i.e., $x_{1}, x_{2} \in V(B)-y$.

Then $B^{2}$ has an $x y$-hamiltonian path $P_{1}$ containing different edges $x_{1} y_{1}, x_{2} y_{2}$ of $E(G)$ for certain $y_{1}, y_{2}$ by Theorem 7 or by Theorem 8 if $x_{1}=x$ or $x_{2}=x$; and $(G-B)^{2}$ has an $x y$-hamiltonian path $P_{2}$ containing different edges $x_{3} y_{3}, x_{4} y_{4}$ of $E(G)$ for certain $y_{3}, y_{4}$ by Lemma 9. Now $P_{1} \cup P_{2}$ is a required hamiltonian cycle in $G^{2}$, a contradiction. Note that $x_{3}, x_{4} \in V\left(B^{\prime}\right)-y^{\prime}$ where $y^{\prime} \in V\left(B^{\prime}\right)$ is a cutvertex of $G-f$, otherwise we can use $B^{\prime}$ instead of $B$ and $x_{3}$ or $x_{4}$ instead of $x_{1}$ or $x_{2}$ (see Subcase 1.2 or Subcase 1.3 below).

Subcase 1.2: $|X \cap(V(B)-y)|=1$; i.e., $x_{1} \in V(B)-y$ and $x_{2} \notin V(B)-y$.

(1.2.1) Assume that $x_{2}, x_{3}, x_{4}$ are not inner vertices of $G$ in the same block of $G-B$. We proceed very similar as in Subcase 1.1; we use only the strong $\mathcal{F}_{3}$ property in $B$, and $G-B$ is a non-trivial blockchain. Hence we can apply Lemma 9 except if $x=x_{1}$, some $x_{i}=y$ for $i \in\{2,3,4\}$, say $i=2$, and $x_{3}, x_{4}$ are inner vertices in the same endblock of $G-B$ which also contains $x_{2}$.

If $x=x_{1}, x_{2}=y$, and $x_{3}, x_{4}$ are inner vertices in the same endblock of $G-B$ which also contains $x_{2}$, then $B^{2}$ has an $x_{2} x_{1}$-hamiltonian path $P_{1}$ containing different edges $x_{2} y_{2}, u v$ of $E(G)$ for certain $y_{2}, u, v$ by Theorem 8 , and $(G-B)^{2}$ has an $x_{2} x_{1}$-hamiltonian path $P_{2}$ containing different edges $x_{1} x^{\prime}, x_{3} y_{3}, x_{4} y_{4}$ of $E(G)$ for certain $y_{3}, y_{4}$ by Lemma 9. Again, $P_{1} \cup P_{2}$ is a required hamiltonian cycle in $G^{2}$, a contradiction.

(1.2.2) Assume that $x_{2}, x_{3}, x_{4}$ are inner vertices of $G$ in the same block $B^{*}$ of $G-B$. 
Clearly, $B^{2}$ contains a hamiltonian cycle $H_{B}$ containing 3 different edges $y^{\prime} y, x_{1}^{\prime} x_{1}, x^{\prime \prime} x$ of $E(B)$ for certain vertices $y^{\prime}, x_{1}^{\prime}, x^{\prime \prime}$ by Theorem 7 (starting with a corresponding $\mathcal{F}_{4} x^{\prime \prime} x$-hamiltonian path in $B^{2}$ ) if $x \neq x_{1}$, and $y^{\prime} y, x_{1}^{\prime} x, x^{\prime \prime} x$ of $E(B)$ for certain vertices $y^{\prime}, x_{1}^{\prime}, x^{\prime \prime}$ by Theorem 5 if $x=x_{1}$.

Let $G_{1}$ be the component of $G-B^{*}-x x^{\prime}$ containing $B$ and $y^{*}=V\left(B^{*}\right) \cap V\left(G_{1}\right)$. Note that $G_{1}$ is a trivial or non-trivial blockchain.

(a) If $y^{*}=y$, then $G_{1}=B$ and we set $H_{G_{1}}=H_{B}$ (see above).

(b) If $y^{*} \neq y$, then either $G_{1}-B=y^{*} y$ or $\left(G_{1}-B\right)^{2}$ contains a hamiltonian cycle $C$ containing edges $y_{1}^{*} y^{*}, y^{\prime \prime} y$ of $E\left(G_{1}-B\right)$ for certain $y_{1}^{*}, y^{\prime \prime}$ by applying Theorem 5 or Theorem 6 .

Now we set

$$
H_{G_{1}}=\left(H_{B}-y^{\prime} y\right) \cup y^{\prime} y^{*}
$$

and $y_{1}^{*}=y$ if $G_{1}-B=y^{*} y$; and

$$
H_{G_{1}}=\left(H_{B} \cup C-\left\{y^{\prime} y, y^{\prime \prime} y\right\}\right) \cup y^{\prime} y^{\prime \prime}
$$

if $G_{1}-B \neq y^{*} y$.

Note that the edge $y_{1}^{*} y^{*} \in E\left(G_{1}\right)$ is contained in $H_{G_{1}}$ in both cases.

Clearly, $\left|V\left(B^{*}\right)\right|+\left|E\left(B^{*}\right)\right|<|V(G)|+|E(G)|$. Hence $\left(B^{*}\right)^{2}$ contains a hamiltonian cycle $H_{B^{*}}$ containing four different edges $y_{2}^{*} y^{*}, x_{2} x_{2}^{\prime}, x_{3} x_{3}^{\prime}, x_{4} x_{4}^{\prime}$ of $E\left(B^{*}\right)$ for certain vertices $y_{2}^{*}, x_{i}^{\prime}, i=2,3,4$.

Let $z \in V\left(B^{*}\right)$ be the cutvertex of $G-x^{\prime} x$ different from $y^{*}$.

(A) $x^{\prime}=z$. Then

$$
\left(H_{G_{1}} \cup H_{B^{*}}-\left\{y_{2}^{*} y^{*}, y_{1}^{*} y^{*}\right\}\right) \cup\left\{y_{1}^{*} y_{2}^{*}\right\}
$$

is a required hamiltonian cycle in $G^{2}$ containing four different edges $x_{i} x_{i}^{\prime}$, of $E(G), i=1,2,3,4$, a contradiction.

(B) $x^{\prime} \neq z$

If $d_{G-B^{*}}(z)=1$, then we set $G_{2}=G-G_{1}-B^{*}-z_{1} z$ where $z_{1}$ is the unique neighbor of $z$ in $G-B^{*}$; otherwise we set $G_{2}=G-G_{1}-B^{*}$. Note that $G_{2}$ is a trivial or non-trivial blockchain and $G_{2}=x^{\prime} x$ is not possible because of $d_{G}\left(x^{\prime}\right)>2$.

We apply Theorem 6 such that either $\left(G_{2}\right)^{2}$ contains a hamiltonian cycle $H_{G_{2}}$ with $x^{\prime} x \in E\left(H_{G_{2}}\right)$ if $z \notin V\left(G_{2}\right)$, or $\left(G_{2}\right)^{2}$ contains a hamiltonian cycle $H$ containing the edge $x^{\prime} x$ and different edges $z_{1} z, z_{2} z$ of $G_{1}$ for certain $z_{1}, z_{2}$ if $z \in V\left(G_{2}\right)$. In the latter case we set $H_{G_{2}}=\left(H-\left\{z_{1} z, z_{2} z\right\}\right) \cup z_{1} z_{2}$. Then

$$
\left(H_{G_{1}} \cup H_{G_{2}} \cup H_{B^{*}}-\left\{y_{2}^{*} y^{*}, y_{1}^{*} y^{*}, x^{\prime} x, x^{\prime \prime} x\right\}\right) \cup\left\{y_{1}^{*} y_{2}^{*}, x^{\prime \prime} x^{\prime}\right\}
$$

is again a hamiltonian cycle in $G^{2}$ containing four different edges $x_{i} x_{i}^{\prime}$ of $E(G), i=1,2,3,4$, a contradiction.

Subcase 1.3: $|X \cap(V(B)-y)|=0$; i.e., $x_{1}, x_{2} \notin V(B)-y$.

Let $G_{1}$ be a graph which arises from $G$ by replacing $B$ with a path $p$ of length 3 , say $p=x, a, b, y$. Then $\left|V\left(G_{1}\right)\right|+\left|E\left(G_{1}\right)\right|<$ $|V(G)|+|E(G)|$ since $B$ is not a triangle because $G$ is edge-critical. Hence $\left(G_{1}\right)^{2}$ contains a hamiltonian cycle $H_{1}$ containing four different edges $x_{i} y_{i}$ of $E\left(G_{1}\right)$ for certain vertices $y_{i}, i=1,2,3,4$, and as many edges as possible of $G_{1}$.

In the following we shall proceed in a manner very similar to the proof in [6] that the square of a 2-block is hamiltonian. However, in order to avoid total dependence of the reader on the knowledge or study of [6], we shall describe and partially repeat the procedure employed in that paper. In particular, we shall quote the cases with the numbering of [6].

This yields the consideration of 13 cases on how the hamiltonian cycle $H_{1}$ traverses vertices of the path $p$. As in [6], Cases 3, Case 4, Case 12, and Case 13 are contradictory to the maximality of the number of edges of $G_{1}$ belonging to $H_{1}$; and Case 6 can be reduced to Case 10, Case 8 to Case 7, Case 10 to Case 9 and Case 11 to Case 5 . Note that by the reductions we preserve the existence of the edges $x_{i} y_{i}$ even if $x_{i} \in\left\{x^{\prime}, y\right\}$ for $i \in\{1,2,3,4\}$.

The remaining 5 cases are (using the labeling of vertices $x^{\prime}, x, a, b, y$ instead of $x, w, a, b, v$ in [6]):

Case $1 . H_{1}=\ldots, x, a, b, y, \ldots$

Case 2. $H_{1}=\ldots, x, a, b, y^{\prime}, \ldots$

Case 5. $H_{1}=\ldots, x^{\prime}, a, b, x, \ldots$

Case 7. $H_{1}=\ldots, x^{\prime}, a, y, \ldots, y^{\prime}, b, x$

Case 9. $H_{1}=\ldots, x^{\prime}, a, y, b, x \ldots$;

and $y^{\prime} y$ is an edge of $G$.

In order to extend $H_{1}$ to $H$ in $G^{2}$ in these five cases with $H$ having the required property, one can proceed in the same way as it has been done in [6]. However, we deem it necessary to show explicitly that no problems arise under the stronger condition of this theorem (similarly as in [7]).

Case 1. By Theorem $8, B^{2}$ has an $x y$-hamiltonian path $P$ starting with an edge $y y^{*}$ of $E(B)$ and containing an edge $u v$ of $B$ for certain vertices $u, v$. Replace in $H_{1}$ the path $p$ with a hamiltonian path $P$ and we get a hamiltonian cycle $H$ as required.

Case 2. Take $P$ as in Case 1 and replace in $H_{1}$ the path $x, a, b, y^{\prime}$ with $\left(P-y y^{*}\right) \cup y^{\prime} y^{*}$ and again we get a hamiltonian cycle $H$ as required. Note that $H$ contains all edges of $G$ belonging to $H_{1}$. 
Case 5. By Theorem $5, B^{2}$ contains a hamiltonian cycle $H_{B}$ such that both edges of $H_{B}$ incident to $y$ (say $y y^{*}, y y^{* *}$ ) are in $B$ and at least one of the edges of $H_{B}$ incident to $x$ (say $x x^{*}$ ) is in $B$. We set

$$
H^{*}=\left(H_{B}-\left\{y y^{*}, y y^{* *}\right\}\right) \cup y^{*} y^{* *}
$$

which does not contain $y$, and replace in $H_{1}$ the path $x^{\prime}, a, b, x$ with $\left(H^{*}-x x^{*}\right) \cup x^{\prime} x^{*}$, thus obtaining a hamiltonian cycle $H$ in $G^{2}$ which has the same behavior in all vertices of $G_{1}-\{a, b\} \subset G$ as $H_{1}$.

Case 7. Take $H_{B}$ as in Case 5 and replace in $H_{1}$ the path $x^{\prime}, a, y$ with the path $P_{1} \cup x^{*} x^{\prime}$ where $P_{1} \subset H_{B}$ is the path from $y$ to $x^{*}$ and does not contain $x$; and replace in $H_{1}$ the path $y^{\prime}, b, x$ with the path $P_{2} \cup y^{\prime} t$ where $t \in\left\{y^{*}, y^{* *}\right\}$ and $P_{2} \subset H_{B}$ is the path from $x$ to $t$ and does not contain any of $y, x^{*}$. Again we get a hamiltonian cycle $H$ as required.

Case 9. Take $H_{B}$ as in Case 5 and replace in $H_{1}$ the path $x^{\prime}, a, y, b, x$ with $\left(H_{B}-x x^{*}\right) \cup x^{\prime} x^{*}$, thus obtaining a hamiltonian cycle $H$ in $G^{2}$ which has the same behavior in all vertices of $G_{1}-\{a, b, y\} \subset G$ as $H_{1}$ and both edges of $H$ incident to $y$ are in $G$.

In all cases we obtained a hamiltonian cycle $H$ in $G^{2}$ containing four different edges $x_{i} x_{i}^{\prime}$, of $E(G)$ (in most cases we have $x_{i}^{\prime}=y_{i}$; see the first paragraph of this subcase 1.3$), i=1,2,3,4$, a contradiction.

Case 2. $|D(G)|=0$. That is, $G$ is a $D T$-graph.

(a) Suppose $N\left(x_{i}\right) \subseteq V_{2}(G)$ for every $i=1,2,3,4$.

Set $W^{\prime}=\left\{x_{1}, x_{2}, x_{3}, x_{4}\right\}$ and let $K$ be a $W^{\prime}$-maximal cycle in $G$. Observe that $|V(K)| \geq 4$ since an edge-critical block on at least 4 vertices cannot contain a triangle.

If $\left|W^{\prime} \cap V(K)\right|=4$, then we choose $x_{5}$ arbitrary in $V(G)-W^{\prime}$. If $\left|W^{\prime} \cap V(K)\right|=3$, then we choose $x_{5}$ arbitrary in $V(K)-W^{\prime}$. If $\left|W^{\prime} \cap V(K)\right|=2$, then we choose an arbitrary 2-valent vertex $x_{5}$ in $V(K)-W^{\prime}$ which exists because all neighbors of $x_{i}$ are 2-valent.

We set $W=W^{\prime} \cup\left\{x_{5}\right\}$. Then $K$ is $W$-sound in $G$ unless $|W \cap V(K)|=3$ and forbidden situation (2) in Definition 10 arises. That is, without loss of generality $x_{1}, x_{2} \in V(K)$ and there exist $W$-separated $K$-to- $K$ blockchains $P, Q$ based on $x_{i}, i \in\{1,2\}, P \cap Q=x_{i}$, and paths $p, q$ in $P, Q$, respectively, such that there is a subsequence $x_{i}, w^{\prime}, L(p), L(q), w^{\prime \prime}, x_{i}$, where $\left\{w^{\prime}, w^{\prime \prime}\right\}=\left\{x_{3-i}, x_{5}\right\}$ and $x_{3}, x_{4} \in V(p) \cup V(q)$. Then there is a cycle $K^{\prime}$ containing $x_{i}, x_{3}, x_{4}$, a contradiction to the $W^{\prime}$-maximality of $K$.

By Theorem 11, $G$ contains an EPS-graph $S=E \cup P$ such that $K \subseteq E$ and $d_{P}(w) \leq 1$ for every $w \in W$. If there is no adjacent pair $x_{i}, x_{j}$ for $i, j \in\{1,2,3,4\}$, we use $S$ and an algorithm in [5] to obtain a hamiltonian cycle in $G^{2}$ with the required properties, a contradiction. However, if there is an adjacent pair, say $x_{1}, x_{2}$, then $d_{G}\left(x_{1}\right)=d_{G}\left(x_{2}\right)=2$ and $d_{P}\left(x_{1}\right)=d_{P}\left(x_{2}\right)=0$ and we can proceed with the cycle $K$ containing $x_{1}, x_{2}, x_{3}$ to obtain a required hamiltonian cycle in $G^{2}$ as before, a contradiction.

(b) Without loss of generality suppose that $N\left(x_{4}\right) \nsubseteq V_{2}(G)$.

Hence $\operatorname{deg}_{G}\left(x_{4}\right)=2$. Let $P_{4}=y_{4} x_{4} z_{1} \ldots z_{k}$ be a unique path in $G$ such that $d_{G}\left(y_{4}\right)>2, d_{G}\left(z_{k}\right)>2$ and $d_{G}\left(z_{i}\right)=2$, for $i=1,2, \ldots, k-1$. We set $G^{-}=G-\left\{x_{4}, z_{1}, \ldots, z_{k-1}\right\}$, where $z_{0}=x_{4}$ if $k=1$.

(b1) Assume that $G^{-}$is 2-connected.

If $x_{i} \in V\left(G^{-}\right)-\left\{y_{4}, z_{k}\right\}$ for $i=1,2,3$, then $|V(G)|+|E(G)|>\left|V\left(G^{-}\right)\right|+\left|E\left(G^{-}\right)\right|$and hence $\left(G^{-}\right)^{2}$ has a hamiltonian cycle $H^{-}$containing different edges $x_{i} y_{i}, z_{k} w_{4} \in E(G), i=1,2$, 3. It is easy to see that we can extend $H^{-}$to a hamiltonian cycle $H$ in $G^{2}$ such that $H$ contains edges $x_{i} y_{i}, x_{4} z_{1}$, for $i=1,2,3$, a contradiction.

Suppose $x_{3} \notin V\left(G^{-}\right)-\left\{y_{4}, z_{k}\right\}$. If $\left\{\left\{x_{1}, x_{2}, x_{3}\right\} \cap\left\{y_{4}, z_{k}\right\} \neq \emptyset\right\}$, then without loss of generality $x_{3} \in\left\{y_{4}, z_{k}\right\}$. By Theorem 7 or Theorem $8,\left(G^{-}\right)^{2}$ contains a $y_{4} z_{k}$-hamiltonian path $P^{-}$and $P^{-}$contains distinct edges $x_{i} y_{i}$ of $G$ if $x_{i} \in V\left(G^{-}\right)$for $i=1,2$. Then $P^{-} \cup P_{4}$ is a hamiltonian cycle in $G^{2}$ with the required properties, a contradiction.

(b2) Assume that $G^{-}$is not 2-connected.

Then $G^{-}$is a non-trivial blockchain with $y_{4}, z_{k}$ in distinct endblocks and $y_{4}, z_{k}$ are not cutvertices.

Assume not all $x_{1}, x_{2}, x_{3}$ are inner vertices in the same block. Then we apply Lemma 9 to get a $y_{4} z_{k}$-hamiltonian path $P^{-}$in $\left(G^{-}\right)^{2}$ with distinct edges $x_{i} y_{i} \in E\left(G^{-}\right), i=1,2$, 3. Note than $x_{i}$ could be $y_{4}$ or $z_{k}$. Then again $P^{-} \cup P_{4}$ is a hamiltonian cycle in $G^{2}$ with the required properties, a contradiction.

Now assume that $x_{1}, x_{2}, x_{3}$ are inner vertices in the same block $B$. Then there exists an end block $B^{*}$ of $G^{-}$such that $x_{i} \notin V\left(B^{*}\right), i=1,2,3$. A graph $G^{\prime}$ arises from $G$ by the replacement of $B^{*}$ by a path $p$ of length 3 . Hence $|V(G)|+|E(G)|>\left|V\left(G^{\prime}\right)\right|+\left|E\left(G^{\prime}\right)\right|$ and we denote by $H^{\prime}$ a hamiltonian cycle in $\left(G^{\prime}\right)^{2}$ containing edges $x_{i} w_{i}, i=1,2,3,4$, and as many edges of $G^{\prime}$ as possible.

We proceed in the same manner as in Subcase 1.3 (note that in this case none of $x_{i}, i=1,2,3,4$, is on $p$ ) to get a hamiltonian cycle in $G^{2}$ with required properties, a contradiction.

Finally we want to show that Theorem 2 is best possible, i.e., we construct an infinite family of graphs which do not satisfy the $\mathcal{H}_{5}$ property. For this purpose start with an arbitrary 2-block $G$ and fix different vertices $x_{1}, x_{2} \in V(G)$.

Define

$$
H=G \cup\left\{y_{1}, y_{2}, \ldots, y_{t} ; t \geq 3\right\} \cup\left\{x_{i} y_{j}: 1 \leq i \leq 2,1 \leq j \leq t\right\},
$$

where $\left\{y_{1}, \ldots, y_{t}\right\} \cap V(G)=\emptyset$. Then $H$ is a 2-block. However, $H$ does not have the $\mathcal{H}_{5}$ property: indeed, there is no hamiltonian cycle $C$ in $H^{2}$ containing edges of $H$ incident to $x_{1}, x_{2}, y_{1}, y_{2}, y_{3}$ because of the neighbors of $y_{1}, y_{2}, y_{3}$ in $H$ which are $x_{1}$ and $x_{2}$ only; that is $x_{1}$ or $x_{2}$ would be incident to three edges of $C \cap H$, which is impossible. 


\section{Conclusion}

We introduced the concept of the $\mathcal{H}_{k}$ property and proved that every 2-block has the $\mathcal{H}_{4}$ property but not the $\mathcal{H}_{5}$ property in general. Similarly in [8] it is proved that every 2-block has the $\mathcal{F}_{4}$ property but not the $\mathcal{F}_{5}$ property in general. Moreover, a 2-block $G$ having the $\mathcal{F}_{k}$ property implies that $G$ has the $\mathcal{H}_{k-1}$ property for $k=3,4, \ldots$. Hence we conclude that Theorems 2 and 7 are best possible with respect to hamiltonicity and hamiltonian connectedness in the square of a 2-block.

\section{Declaration of competing interest}

The authors declare that they have no known competing financial interests or personal relationships that could have appeared to influence the work reported in this paper.

\section{Acknowledgments}

This publication was supported by the project L01506 of the Czech Ministry of Education, Youth and Sports, and by FWF, Austria project P27615-N25.

\section{References}

[1] S. Alstrup, A. Georgakopoulos, E. Rotenberg, C. Thomassen, Carsten, A Hamiltonian cycle in the square of a 2-connected graph in linear time, in: Proceedings of the Twenty-Ninth Annual ACM-SIAM Symposium on Discrete Algorithms, SIAM, Philadelphia, PA, 2018, pp. 1645-1649, (English summary).

[2] J.A. Bondy, U.S.R. Murty, Graph Theory, in: Graduate Texts in Mathematics, vol. 244, Springer, New York, 2008.

[3] G.L. Chia, J. Ekstein, H. Fleischner, Revisiting the Hamiltonian theme in the square of a block: The case of DT-graphs, J. Combin. 9 (1) (2018) $119-161$.

[4] J. Ekstein, Hamiltonian cycles in the square of a graph, Electron. J. Combin. 18 (2011) \#P203.

[5] H. Fleischner, On spanning subgraphs of a connected bridgeless graph and their application to DT-graphs, J. Combin. Theory 16 (1974) 17-28.

[6] H. Fleischner, The square of every two-connected graph is hamiltonian, J. Combin. Theory 16 (1974) $29-34$.

[7] H. Fleischner, In the square of graphs, Hamiltonicity and pancyclicity, hamiltonian connectedness and panconnectedness are equivalent concept, Monatsh. Math. 82 (1976) 125-149.

[8] H. Fleischner, G.L. Chia, Revisiting the hamiltonian theme in the square of a block: The general case, J. Combin. 10 (1) (2019) $163-201$.

[9] H. Fleischner, A.M. Hobbs, Hamiltonian total graphs, Math. Nachr. 68 (1975) 59-82.

[10] A. Georgakopoulos, A short proof of fleischner's theorem, Discrete Math. 309 (2009) 6632-6634.

[11] J. Müttel, D. Rautenbach, A short proof of the versatile version of fleischner's theorem, Discrete Math. 313 (2013) $1929-1933$. 